\title{
A Maxwellian Lower Bound for Solutions to the Boltzmann Equation
}

\section{Ada Pulvirenti ${ }^{1}$, Bernt Wennberg ${ }^{2}$}

${ }^{1}$ Dipartimento di Matematica, Università di Pavia, Via Abbiategrasso 215, 27100 Pavia, Italy. E-mail: ada@dragon.ian.pv.cnr.it

${ }^{2}$ Department of Mathematics, Chalmers University of Technology, 41296 Göteborg, Sweden. E-mail: wennberg@math.chalmers.se

Received: 27 October 1995/Accepted: 3 June 1996

Abstract: We prove that the solution of the spatially homogeneous Boltzmann equation is bounded pointwise from below by a Maxwellian, i.e. a function of the form $c_{1} \exp \left(-c_{2} v^{2}\right)$. This holds for any initial data with bounded mass, energy and entropy, and for any positive time $t \geqq t_{0}$. The constants, $c_{1}$, and $c_{2}$, depend on the mass, energy and entropy of the initial data, and on $t_{0}>0$ only.

A similar result is obtained for the Kac caricature of the Boltzmann equation, where the proof is easier.

\section{Introduction}

We consider the spatially homogeneous Boltzmann equation,

$$
\partial_{t} f=Q(f, f),
$$

where $f=f(v, t), v \in \mathbb{R}^{3}$, is a non-negative function which gives the velocity distribution of a (spatially homogeneous) dilute gas. The bilinear operator $Q$ is the so-called collision operator. It is given by

$$
Q(f, g)(v)=\iint\left(f\left(v^{\prime}\right) g\left(v_{1}^{\prime}\right)-f(v) g\left(v_{1}\right)\right) B\left(\left|v-v_{1}\right|, \theta\right) d \omega d v_{1},
$$

where $v^{\prime}$ and $v_{1}^{\prime}$ are the velocities after the collision of two particles which had the velocities $v$ and $v_{1}$ before the collision. The velocities before and after a collision are related by

$$
\begin{aligned}
& v^{\prime}=v+\left[\left(v-v_{1}\right) \cdot \omega\right] \omega, \\
& v_{1}^{\prime}=v_{1}-\left[\left(v-v_{1}\right) \cdot \omega\right] \omega .
\end{aligned}
$$

The collision operator $Q$ has the form (1.2) for all monatomic gases. The exact form of the interaction between the particles is given by the collision kernel, $B$. In this paper we deal only with the so-called hard potentials with an angular cut-off. In this case,

$$
B\left(\left|v-v_{1}\right|, \theta\right)=h(\theta)\left|v-v_{1}\right|^{\beta},
$$


with $0 \leqq \beta \leqq 1$, and $\int_{0}^{\pi / 2} h(\theta) d \theta<\infty$; elastic spheres are modelled by $\beta=1$, and $h(\theta)=\cos \theta$. The cut-off potentials simplify the mathematical treatment of the equation because they make it possible to write the collision operator as the difference of two positive terms, $Q^{+}(f, f)$ and $Q^{-}(f, f) \equiv f L f$, which are defined as the terms containing the velocities after and before a collision respectively; these are known as the gain term and the loss term.

The mathematical theory for the space independent Boltzmann equation is by now rather complete. It is known that the equation is well posed, and that the unique solution conserves mass, momentum and energy:

$$
\int_{\mathbb{R}^{3}} f(v, t) \phi_{i} d v=\int_{\mathbb{R}^{3}} f_{0}(v) \phi_{i} d v .
$$

Here $\phi_{0}=1, \phi_{i}=v^{i}, i=1,2,3,\left(v^{i}\right.$ are the components of $\left.v\right)$, and $\phi_{4}=|v|^{2}$. Since mass is conserved, there is no loss of generality in assuming that this is normalized to one.

Moreover, the entropy is a decreasing function of time:

$$
\int_{\mathbb{R}^{3}} f(v, t) \log f(v, t) d v \leqq \int_{\mathbb{R}^{3}} f_{0}(v) \log f_{0}(v) d v .
$$

The entropy is minimized by the so-called Maxwellians, which are functions of the form $a \exp \left(-(v-b)^{2} / c\right)$; these are the only equilibrium solutions, and the solutions of (1.1) converge strongly to the unique Maxwellian which has the same mass, momentum and energy as the initial data $f_{0}$. Initial data for which all these quantities are bounded are said to satisfy the natural bounds. For a more general introduction to the physical background as well as to the mathematical theory, we refer to $[\mathrm{Ce}]$.

The first mathematically rigorous results dealing with the Boltzmann equation are due to Carleman ([Ca1, $\mathrm{Ca} 2])$. He considered a gas of elastic spheres, and proved that the above properties hold, under the additional condition that the initial data be Hölder continuous, and satisfy a moment condition,

$$
\int_{\mathbb{R}^{3}} f_{0}(v)|v|^{6} d v<\infty .
$$

In the course of the proof he also proved that the solutions must be bounded from below by exponential functions, $c \exp \left(-|v|^{2+\varepsilon}\right)$, with $\varepsilon>0$ arbitrarily small. Lower bounds of this type are essential for determining the rate of entropy dissipation,

$$
\frac{d}{d t} \int_{\mathbb{R}^{3}} f(v, t) \log f(v, t) d v=\int_{\mathbb{R}^{3}} Q(f, f)(v) \log f(v, t) d v .
$$

They are used by Carleman for this purpose, and more recently Carlen and Carvalho (see e.g. [C-C]) have made a detailed study of the entropy production rate, in which also lower bounds play an important role. A different approach was taken by Desvillettes [D1], and later by Wennberg [W], but also in those papers, the solutions were assumed to be bounded pointwise from below.

In this paper we prove that the result of Carleman holds for all realistic initial data (i.e. with bounded mass energy and entropy), and that the exponential can be 
replaced by a Maxwellian $(\varepsilon=0)$. This improvement may seem a minor one, but in fact it has a direct application for example in the papers by Carlen and Carvalho. In addition, it is likely that precise estimates for the spatially homogeneous Boltzmann equation may be useful in obtaining results for the more interesting case of the full, space dependent equation.

We begin by stating the main result, which will be proved in Sect. 3 .

Theorem 1.1. Let $f_{0}(v) \geqq 0$ be given with $\int f_{0}(v)\left(1+|v|^{2}+\log f_{0}(v)\right) d v<\infty$, and let $f(v, t)$ be the solution of the Boltzmann equation, (1.1), for hard potentials, and with $f_{0}(v)$ as initial data. Then, for any positive time, $f(v, t)$ is bounded pointwise from below by a Maxwellian,

$$
f(v, t) \geqq C_{1} e^{-C_{2}|v|^{2}} .
$$

The constants $C_{1}$ and $C_{2}$ depend on the mass, energy and entropy of the initial data and on $t>0$, as well as on the collision kernel. In general the constants improve as $t$ increases: $C_{1}$ and $C_{2}$ can be chosen uniformly for all $t>t_{0}$, where $t_{0}$ is any positive time.

Apart from the estimates by Carleman, we are not aware of other precise pointwise lower bounds for the Boltzmann equation. However, Bobylev has obtained an estimate of the average behaviour at large velocities, of isotropic solutions in the case of Maxwellian molecules (cf. [Bo]). In a previous paper [P-W], the estimate of Carleman was obtained for general initial data as in (1.1), though only for Maxwellian molecules $(\beta=0$ in (1.3)).

The proof of Theorem 1.1 consists of two main parts. First we prove that at any positive time, $t_{0}$, there is a ball, $|v-\bar{v}|<\delta_{0}$, such that in this ball, $f\left(v, t_{0}\right)>\varepsilon_{0}$. It is important that $\varepsilon_{0}$ and $\delta_{0}$ depend only on the same quantities as do the constants in the theorem. Next we prove that once this estimate has been established, it follows that at $t_{1}>t_{0}, f\left(v, t_{0}\right)>\varepsilon_{1}$ for $|v-\bar{v}|<\delta_{1}$, where $\delta_{1}>\delta_{0}$. The proof is then completed by an iteration procedure.

Section 2 is devoted to the Kac equation (see [K]), which is a model of the Boltzmann equation. The Kac equation can be written as (1.1), but in this case $v \in \mathbb{R}$, and the lower dimension makes the collision operator much simpler than the real one. In spite of this simplification, the equation is in many ways similar to the Boltzmann equation, and the main result in Sect. 2 is a direct analogue of Theorem 1.1, and the spirit of the proof is the same. However, it is slightly less complicated, and we hope that by including it in the paper, the ideas will become more clear. Of course, the Kac equation is interesting in its own right, and it has been studied in several papers recently (see [D2, G-T, G-P]), which gives still another motivation for including a separate section on this matter.

\section{The Kac Equation}

The Kac equation is a simplified Boltzmann-like equation, which describes the evolution of a one-dimensional gas, in which the collision operator $Q(f, f)$, is given by

$$
\int_{-\infty}^{\infty} \int_{-\pi}^{\pi} \frac{1}{2 \pi}\left(f\left(v^{\prime}\right) f\left(w^{\prime}\right)-f(v) f(w)\right) d \theta d w
$$


All possible collisions are now two-dimensional rotations:

$$
\begin{gathered}
v^{\prime}=v \cos \theta-w \sin \theta, \\
w^{\prime}=v \sin \theta+w \cos \theta,
\end{gathered}
$$

which preserve the energy, but not the momentum and, in the original form introduced by $\mathrm{Kac}[\mathrm{K}]$, are considered equiprobable. Although it is possible to generalize the collision operator by replacing the constant factor $1 / 2 \pi$ by a rate function depending on $\theta$ (as in the references given above), we will consider only the simpler case (2.1), since, before passing to the Boltzmann equation, we want to explain our method for constructing lower bounds in an easier, but quite reasonable case. In fact the Kac equation has many properties in common with the usual Boltzmann equation: the solutions conserve mass, $\int_{\mathbf{R}} f(v, t) d v$, and energy, $\int_{\mathbf{R}} f(v, t) v^{2} d v$ and the corresponding $H$-functional, $H(f)=\int_{\mathbf{R}} f(v) \log f(v) d v$ decreases in time. Momentum, however, is conserved only if it vanishes. Thus the following are natural conditions for the initial data

$$
\int_{\mathbb{R}} f_{0}(v) d v=1, \quad \int_{\mathbb{R}} v f_{0}(v) d v=0, \quad \int_{\mathbb{R}} v^{2} f_{0}(v) d v=E<\infty .
$$

We also require that the entropy is bounded initially; in fact, in this section we make the stronger assumption that the Linnik functional is bounded. The Linnik functional is defined as

$$
L\left(f_{0}\right) \equiv \int_{\mathbf{R}} \frac{\left[f_{0}^{\prime}(v)\right]^{2}}{f_{0}(v)} d v=L_{0}<\infty .
$$

This functional is decreasing for solutions of the Kac equation, and it is minimized by the Maxwellians, just like the usual entropy. It is convenient to use here because it gives estimates of the regularity of the solutions.

With the initial data normalized as in (2.2) the Kac equation takes the form

$$
\frac{\partial f}{\partial t}=Q^{+}(f, f)(v)-f(v)
$$

(the gain term $Q^{+}$is defined in the obvious way by $(2.1)$ ) and the solution can be represented as a so-called Wild sum (cf. eg. [M]):

$$
f(v, t)=e^{-t} f_{0}(v)+e^{-t} \sum_{n=1}^{\infty}\left(1-e^{-t}\right)^{n} f_{n}(v)
$$

The terms $f_{n}$ are computed recursively from the initial data,

$$
\begin{aligned}
f_{0}(v) & =f(v, 0) \\
f_{n+1}(v) & =\frac{1}{n+1} \sum_{k=0}^{n} Q^{+}\left(f_{k}, f_{n-k}\right)(v) .
\end{aligned}
$$

We begin by giving some properties of the terms of the Wild sum, and of the solutions of the Kac equation 
Lemma 2.1. If $f_{0}$ satisfies conditions (2.2), (2.3), then every term of the Wild sum satisfies the same conditions, i.e.

$$
\int_{\mathbb{R}} f_{n}(v) d v=1, \quad \int_{\mathbb{R}} v^{2} f_{n}(v) d v=E, \quad L\left(f_{n}\right) \leqq L\left(f_{0}\right) .
$$

Moreover

$$
\left\|f_{n}\right\|_{\infty} \leqq\left[L\left(f_{n}\right)\right]^{1 / 2} .
$$

Proof. See for example [M] and [G-T].

A direct consequence of the boundedness of $L\left(f_{0}\right)$, is the following Hölder estimate.

Lemma 2.2. If $L\left(f_{0}\right)<\infty$, then

$$
\left|f\left(v_{1}\right)-f\left(v_{2}\right)\right| \leqq[L(f)]^{3 / 4}\left|v_{1}-v_{2}\right|^{1 / 2} .
$$

Proof.

$$
\begin{aligned}
\left|f\left(v_{1}\right)-f\left(v_{2}\right)\right| & \leqq \int_{v_{1}}^{v_{2}}\left|f^{\prime}(w)\right| d w \leqq\left[\int_{v_{1}}^{v_{2}} \frac{f^{\prime}(w)^{2}}{f(w)} d w\right]^{1 / 2}\left[\int_{v_{1}}^{v_{2}} f(w) d w\right]^{1 / 2} \\
& \leqq[L(f)]^{1 / 2}[L(f)]^{1 / 4}\left|v_{1}-v_{2}\right|^{1 / 2} \\
& =[L(f)]^{3 / 4}\left|v_{1}-v_{2}\right|^{1 / 2} \cdot
\end{aligned}
$$

Next we prove that the solution $f$ is strictly positive in an interval near the origin.

Lemma 2.3. If $f$ and $g$ satisfy conditions (2.6) and (2.7) then $Q^{+}(f, g)$ satisfies

$$
\begin{aligned}
& Q^{+}(f, g)(0)>\frac{1}{3 \pi \sqrt{3 E}}, \\
& Q^{+}(f, g)(v)>\frac{1}{6 \pi \sqrt{3 E}}, \text { for }|v|<\frac{1}{C_{E, L\left(f_{0}\right)}} .
\end{aligned}
$$

Moreover, if $f(v, t)$ is the solution of $(2.4)$ with initial data $f_{0}(v)$, and if $f_{0}$ satisfies $(2.2,2.3)$, then for any positive time $t_{0}$,

$$
f\left(v, t_{0}\right)>e^{-t_{0}}\left(1-e^{-t_{0}}\right) \frac{1}{6 \pi \sqrt{3 E}}, \quad \text { for }|v|<\left(C_{E, L\left(f_{0}\right)}\right)^{-1} .
$$

Proof. By a change of variables,

$$
\begin{aligned}
& \rho=\sqrt{v^{2}+w^{2}}=\sqrt{v^{\prime 2}+w^{\prime 2}}, \\
& v^{\prime}=\rho \cos \varphi, \quad w^{\prime}=\rho \sin \varphi,
\end{aligned}
$$


the gain term $Q^{+}$becomes

$$
\begin{aligned}
Q^{+}(f, g)(v) & =\int_{|v|}^{\infty} \int_{-\pi}^{\pi} f(\rho \cos \varphi) g(\rho \sin \varphi) \frac{\rho}{\sqrt{\rho^{2}-v^{2}}} d \varphi d \rho \\
& =\frac{1}{\pi} \int_{-\infty}^{\infty} \int_{\infty}^{\infty} f\left(v^{\prime}\right) g\left(w^{\prime}\right) \frac{\chi_{\left\{v^{\prime 2}+w^{\prime 2}>v^{2}\right\}}}{\sqrt{v^{\prime 2}+w^{\prime 2}-v^{2}}} d v^{\prime} d w^{\prime}
\end{aligned}
$$

Here $\chi$ is the characteristic function of the set $\left\{v^{\prime 2}+w^{\prime 2}>v^{2}\right\}$. On evaluating the integral in (2.10) at $v=0$, we obtain

$$
\begin{aligned}
Q^{+}(f, g)(0)= & \frac{1}{\pi} \int_{-\infty}^{\infty} \int_{-\infty}^{\infty} f\left(v^{\prime}\right) g\left(w^{\prime}\right) \frac{1}{\sqrt{v^{\prime 2}+w^{\prime 2}}} d v^{\prime} d w^{\prime} \\
\geqq & \frac{1}{\sqrt{2} \pi R} \int_{-\infty}^{\infty} \int_{-\infty}^{\infty} f\left(v^{\prime}\right) g\left(w^{\prime}\right) d v^{\prime} d w^{\prime} \\
& -\frac{1}{\sqrt{2} \pi R} \int_{\left|v^{\prime}\right|>R} \int_{-\infty}^{\infty} f\left(v^{\prime}\right) g\left(w^{\prime}\right) d v^{\prime} d w^{\prime} \\
& -\frac{1}{\sqrt{2} \pi R} \int_{-\infty}^{\infty} \int_{\left|w^{\prime}\right|>R} f\left(v^{\prime}\right) g\left(w^{\prime}\right) d v^{\prime} d w^{\prime} \\
\geqq & \frac{1}{\sqrt{2} \pi R}\left[1-\frac{2}{R^{2}} \int_{\left|v^{\prime}\right|>R} v^{\prime 2} f\left(v^{\prime}\right) d v^{\prime}\right] \geqq \frac{1}{\sqrt{2} \pi R}\left[1-\frac{2 E}{R^{2}}\right] .
\end{aligned}
$$

The inequality (2.8) follows by taking $R \geqq \sqrt{6 E}$ and using the Hölder estimate; $C_{E, L\left(f_{0}\right)}=\left(108 \pi^{2} E L_{0}^{3 / 2}\right)^{-1}$. Finally, (2.9) can be obtained by keeping only one term in the Wild sum,

$$
f(v, t)>e^{-t}\left(1-e^{-t}\right) Q^{+}\left(f_{0}, f_{0}\right)(v) .
$$

The following lemma provides an estimate of the support of $Q^{+}(f, f)$ in terms of the support of $f$.

Lemma 2.4. Assume that $f(v)>\varepsilon_{0}$ for $|v| \leqq \delta_{0}$, for some given $\varepsilon_{0}, \delta_{0}>0$. Then

$$
Q^{+}(f, f)(v)>C \varepsilon_{0}^{2} \delta_{0} \gamma^{3 / 2} \text { for }|v|<\delta_{0} \sqrt{2}(1-\gamma) .
$$

Proof. First we note that, since $Q^{+}$is a positive quadratic operator, it is enough to consider the case where $f$ is the characteristic function of the set $\left\{|v| \leqq \delta_{0}\right\}$. An easy computation, that will be carried out in the case of the Boltzmann equation, shows that

$$
Q^{+}(f, f)\left(\delta_{0} v\right)=\delta_{0} Q^{+}\left(f\left(\delta_{0} \cdot\right), f\left(\delta_{0} \cdot\right)\right)(v),
$$

and therefore it is enough to assume that $f(v)=\chi_{\{|v| \leqq 1\}}$. In this case $Q^{+}(f, f)(v)$ is strictly positive for $|v|<\sqrt{2}$; we are now concerned about the asymptotic behaviour 
near $|v|=\sqrt{2}$. For $1<|v|<\sqrt{2}$,

$$
\begin{aligned}
Q^{+}(f, f)(v) & =\frac{4}{\pi} \int_{0}^{\sqrt{2-v^{2}}} \int_{0}^{\pi / 2} \chi_{\left\{\left|v^{\prime}\right| \leqq 1\right\}} \chi_{\left\{\left|w^{\prime}\right| \leqq 1\right\}} d w d \theta \\
& =\frac{4}{\pi} \int_{0}^{\sqrt{2-v^{2}}}\left(\frac{\pi}{2}-2 \arccos \left(\frac{1}{\sqrt{v^{2}+w^{2}}}\right)\right) d w \\
& =\frac{4}{\pi} \int_{0}^{\sqrt{2-v^{2}}} \arcsin \left(\frac{2}{v^{2}+w^{2}}-1\right) d w \\
& \sim \frac{4}{\pi} \int_{0}^{\sqrt{2-v^{2}}}\left(\frac{2}{v^{2}+w^{2}}-1\right) d w=\frac{8}{\pi v} \arctan \frac{\sqrt{2-v^{2}}}{v}-\frac{4}{\pi} \sqrt{2-v^{2}},
\end{aligned}
$$

near $|v|=\sqrt{2}$, and note that the " $\sim$ " could have been replaced by " $\geqq$." By writing $v=\sqrt{2}(1-\gamma)$, and expanding in terms of $\gamma$, we find that

$$
Q^{+}(f, f)(v)=\frac{32}{3 \pi} \gamma^{3 / 2}+\mathcal{O}\left(\gamma^{5 / 2}\right)=\frac{32}{3 \pi}(1-v / \sqrt{2})^{3 / 2}+\mathcal{O}\left((1-v / \sqrt{2})^{5 / 2}\right),
$$

and that gives the result.

We are now ready to prove the main result concerning the Kac equation. Since all terms in the Wild sum are positive, a rough estimate from below can be obtained by using only one of the terms, and therefore

$$
f\left(v, t_{1}\right)>e^{-t_{1}}\left(1-e^{-t_{1}}\right) Q^{+}\left(f_{0}, f_{0}\right)(v)>\frac{t_{1}}{2} Q^{+}\left(f_{0}, f_{0}\right)(v),
$$

if $t_{1}<1 / 3$ (for example). From Lemma 2.3 we know that $f\left(v, t_{0}\right)>\varepsilon_{0}$ if $|v|<\delta_{0}$, and therefore according to Lemma 2.4 ,

$$
f\left(v, t_{0}+t_{1}\right)>\varepsilon_{0}^{2} C \delta_{0} \frac{t_{1} \gamma_{1}^{3 / 2}}{2}, \text { for }|v|<\delta_{0} 2^{1 / 2}\left(1-\gamma_{1}\right) .
$$

Iterating this gives

$$
f\left(v, t_{0}+t_{1}+t_{2}\right)>\left(\varepsilon_{0}^{2} C \delta_{0} \frac{t_{1} \gamma_{1}^{3 / 2}}{2}\right)^{2} C \delta_{0} 2^{1 / 2}\left(1-\gamma_{1}\right)\left(\frac{t_{2} \gamma_{2}^{3 / 2}}{2}\right),
$$

for $|v|<\delta_{0} 2^{2 / 2}\left(1-\gamma_{1}\right)\left(1-\gamma_{2}\right)$, and after $n$ steps,

$$
\begin{aligned}
f\left(v, t_{0}+\cdots+t_{n}\right)> & \varepsilon_{0}^{2^{n}}\left(C \delta_{0}\right)^{2^{n}-1} \cdot\left(2^{1 / 2}\left(1-\gamma_{1}\right)\right)^{2^{n-1-1}} \\
& \cdots \cdot\left(2^{k / 2}\left(1-\gamma_{1}\right) \cdots \cdot\left(1-\gamma_{k}\right)\right)^{2^{n-1-k}} \cdots \cdots \\
& \cdot\left(\frac{t_{1} \gamma_{1}^{3 / 2}}{2}\right)^{2^{n-1}} \cdots\left(\frac{t_{k} \gamma_{k}^{3 / 2}}{2}\right)^{2^{n-k}} \cdots\left(\frac{t_{n} \gamma_{n}^{3 / 2}}{2}\right)^{2^{0}},
\end{aligned}
$$


for $|v|<\delta_{0} 2^{n / 2}\left(1-\gamma_{1}\right) \cdots\left(1-\gamma_{n}\right)$. Good choices for $t_{k}$ and $\gamma_{k}$ are $t_{k}=t_{0}^{k}$ and $\gamma_{k}=\gamma_{0}^{k}$, where the constants $t_{0}$ and $r_{0}$ can be chosen later. The product of the factors $t_{k} \gamma_{k}^{3 / 2}$ is $\left(t_{0} \gamma_{0}^{3 / 2}\right)^{4}$, where

$$
A=2^{n-1} \sum_{k=0}^{n-1}(k+1) 2^{-k}=2^{n}\left(2-(2+n) 2^{-n}\right) \leqq 2^{n+1} .
$$

Assuming that $\gamma_{0}$ is small,

$$
\begin{aligned}
C_{\gamma k} \equiv\left(1-\gamma_{1}\right) \cdots\left(1-\gamma_{k}\right) & =\exp \sum_{j=1}^{k} \log \left(1-\gamma_{0}^{j}\right) \\
& >\exp \left(-2 \sum_{j=1}^{k} \gamma_{0}^{j}\right)>\exp \frac{-2 \gamma_{0}}{1-\gamma_{0}},
\end{aligned}
$$

which is strictly positive (larger than $1 / 2$, say, if $\gamma_{0}$ is sufficiently small). The contribution from the factors of 2 can be accounted for by modifying the constant $C$ that appears in (2.13). Finally we let $t=t_{0}+\sum_{k=1}^{n} t_{k}=t_{0}+t_{0}\left(t_{0}^{n}-1\right) /\left(t_{0}-1\right)$, and then

$$
f(v, t)>\frac{t_{0} \gamma_{0}^{3 / 2}}{\delta_{0} C}\left(C \delta_{0} \varepsilon_{0} t_{0}^{2} \gamma_{0}^{3}\right)^{2^{n}}, \quad \text { for }|v|<\delta_{0} C_{\gamma n} 2^{n / 2} .
$$

Clearly this estimate is interesting only in the interval $\delta_{0} C_{\gamma(n-1)} 2^{(n-1) / 2} \leqq|v|<$ $\delta_{0} C_{\gamma n} 2^{n / 2}$, and therefore $2^{n}<\left(8|v| / \delta_{0}\right)^{2}$, (after replacing $C_{\gamma(n-1)}$ by $1 / 2$ ), and so

$$
f(v, t)>\frac{t_{0} \gamma_{0}^{3 / 2}}{\delta_{0} C}\left(C \delta_{0} \varepsilon_{0} t_{0}^{2} \gamma_{0}^{3}\right)^{\left(8|v| / \delta_{0}\right)^{2}}=C_{1} e^{-C_{2}|v|^{2}}
$$

where

$$
C_{1}=\frac{t_{0} \gamma_{0}^{3 / 2}}{\delta_{0} C}
$$

and

$$
C_{2}=-\left(\frac{8}{\delta_{0}}\right)^{2} \log \left(C \delta_{0} \varepsilon_{0} t_{0}^{2} \gamma_{0}^{3}\right)
$$

Let us remark that all estimates and constants have been obtained only using the mass, the energy and the Linnik functional of the initial datum $f_{0}$, so that the same lower bound holds for all times after $t$. Note also that in this calculation $\varepsilon_{0}=\mathcal{O}\left(t_{0}\right)$.

To summarize, we have proven the following theorem.

Theorem 2.5. Let $f$ be the solution of the Kac equation with initial data $f_{0}$ satisfying conditions (2.2),(2.3). For any there are constants $C_{1}>0$ and $C_{2}>0$ such that

$$
f(v, t) \geqq C_{1} \exp \left(-C_{2}|v|^{2}\right) .
$$

The constants $C_{1}$ and $C_{2}$ depend on the initial mass, energy and Linnik functional and on $t$, and they can only improve as $t$ increases. 


\section{The Boltzmann Equation}

In this section the calculations from Sect. 2 are adapted for the Boltzmann equation. There are essentially two difficulties that must be overcome. First of all, we wish to obtain the result for all the usual collision kernels, and so there is no analogue of the Wild sum that is useful. Instead we use the Duhamel formula to represent the solution, and there are some additional difficulties associated with the fact that the loss term is not bounded in this case. However, the result that will be obtained in the end is exactly the same as the result for the Kac equation, and it is valid for all (hard cutoff) collision kernels that have been studied in e.g. Arkeryd [A1] (and by Di Perna and Lions in [D-L], for the spatially dependent equation).

The second problem is to obtain an analogue of Lemma 2.4. Without making additional hypothesis on the regularity of the initial data, we cannot rely on a Hölder condition to find a ball where the solution is bounded from below by a constant; such an estimate is needed even to start the iteration procedure.

With the notation $G_{t_{1}}^{t_{2}}(v)=\exp \left(-\int_{t_{1}}^{t_{2}} L f(v, \tau) d \tau\right)$, the solution of the Boltzmann equation can be written

$$
f(v, t)=f_{0}(v) G_{0}^{t}(v)+\int_{0}^{t} G_{\tau}^{t}(v) Q^{+}(f(\cdot, \tau), f(\cdot, \tau))(v) d \tau .
$$

A straightforward computation involving the mass, energy and entropy of $f$, shows that there is a constant $c$, such that

$$
L f(v, t) \leqq c\left(1+|v|^{\beta}\right),
$$

and this holds uniformly in time. A similar inequality holds in the reverse direction (cf. [A2]). Hence $G_{t_{1}}^{t_{2}}(v) \geqq \exp \left(-c\left(t_{2}-t_{1}\right)\left(1+|v|^{\beta}\right)\right) \equiv \tilde{G}_{t_{1}}^{t_{2}}(v)$, and with a very crude estimate,

$$
\begin{aligned}
f(v, t) & \geqq \tilde{G}_{0}^{t}(v) f_{0}(v)+\int_{0}^{t} \tilde{G}_{\tau}^{t}(v) Q^{+}(f(\cdot, \tau), f(\cdot, \tau))(v) d \tau \\
& \geqq \int_{0}^{t} \tilde{G}_{\tau}^{t}(v) Q^{+}\left(\tilde{G}_{0}^{\tau} f_{0}, \tilde{G}_{0}^{\tau} f_{0}\right)(v) d \tau .
\end{aligned}
$$

This corresponds to keeping one term of the Wild sum, as in Sect. 2. A similar inequality comes from the analogue of considering two terms of the sum:

$$
\begin{aligned}
f(v, t) & \geqq \tilde{G}_{0}^{t}(v) f_{0}(v)-\int_{0}^{t} \tilde{G}_{\tau}^{t}(v) Q^{+}(f(\cdot, \tau), f(\cdot, \tau))(v) d \tau \\
& \geqq \int_{0}^{t} \tilde{G}_{\tau}^{t}(v) Q^{+}\left(\tilde{G}_{0}^{\tau} f_{0}, \int_{0}^{\tau} \tilde{G}_{\tau_{1}}^{\tau} Q^{+}\left(\tilde{G}_{0}^{\tau_{1}} f_{0}, \tilde{G}_{0}^{\tau_{1}} f_{0}\right) d \tau_{1}\right)(v) d \tau .
\end{aligned}
$$

This estimate will be needed in the proof of Lemma 3.1.

One further simplification of the inequalities (3.1) and (3.2) is possible when it is only necessary to consider $f_{0}$ in a bounded set, $|v|<R$. Then $\tilde{G}_{t_{1}}^{t_{2}}(v)>$ $\exp \left(-c\left(t_{2}-t_{1}\right)\left(1+R^{\beta}\right)\right)$, and then the operator $Q^{+}$can be taken as acting only on $f_{0}$, just as in the case of the Wild sum.

After these preliminary observations, we give the analogues of Lemma 2.3 and Lemma 2.4 . 
Lemma 3.1. Let $f_{0}$ satisfy the natural conditions. There are constants, $R, \delta_{0}, \varepsilon_{0}$, and $\bar{v} \in \mathbb{R}^{3}$, such that $f(v, t)>\varepsilon_{0}$, for all $|v-\bar{v}|<\delta_{0},|\bar{v}|<R$. The constants depend on the mass, energy and entropy of the initial data, and on the kernel of the collision operator. Moreover, $\varepsilon_{0}=\mathcal{O}\left(t^{2}\right)$ for small $t$.

Proof. The idea of the proof is most easily explained for Maxwellian molecules, where the solution of the Boltzmann equation can be written as a Wild sum. The third term of that sum is

$$
Q^{+}\left(Q^{+}\left(f_{0}, f_{0}\right), f_{0}\right),
$$

multiplied by a time dependent factor. The operator $Q^{+}(\cdot, \cdot)$ is given by $(1.2)$, as described in the introduction, but there is an alternative formulation, due to Carleman ([Ca2]), which is also useful in many cases. Rather than parametrizing the sphere $S_{v, v_{1}}$ (the set of all possible $v^{\prime}$ which result from a collision between one particle with velocity $v$ and another one with velocity $v_{1}$, i.e. the sphere which has $\overline{v v_{1}}$ as a diagonal), one considers the set $E_{v, v^{\prime}}$ which is the set of all $v_{1}^{\prime}$ which could be the post-collisional velocity of one particle, if the other post collisional velocity is $v^{\prime}$ and one of the pre-collisional velocities is $v$. It is easy to see that $E_{v, v^{\prime}}$ is the plane, which contains the point $v$, and is perpendicular to $v^{\prime}-v$. Carleman found that in those terms, the gain term can be written

$$
Q^{+}(f, g)(v)=\int_{\mathbf{R}^{3}} f\left(v^{\prime}\right) \frac{1}{\left|v-v^{\prime}\right|^{2-\beta}} \int_{E_{v, v^{\prime}}} g\left(v_{1}^{\prime}\right) \frac{h(\theta)}{(\cos \theta)^{\beta}} d E\left(v_{1}^{\prime}\right) d v^{\prime},
$$

with $d E\left(v_{1}^{\prime}\right)$ denoting the induced Lebesgue measure on $E_{v, v^{\prime}}$.

Suppose now that $f_{0}$ is the sum of two Dirac measures, $\delta_{1}$ and $\delta_{2}$ at $w_{1}$ and $w_{2}$. Then $Q^{+}\left(f_{0}, f_{0}\right)$ is a unit measure concentrated on the sphere $S_{w_{1}, w_{2}}$ (see Fig. 1). Then take $f=f_{0}$ and $g=Q^{+}\left(f_{0}, f_{0}\right)$ in (3.4); if $v$ lies inside $S_{w_{1}, w_{2}}$, and $v^{\prime}=w_{1}$ (or $v^{\prime}=w_{2}$ ), then the plane $E_{v, v^{\prime}}$ intersects $S_{w_{1}, w_{2}}$, and so the term given in (3.3) is non-zero for all $v$ inside the sphere. Clearly similar estimates can be obtained if $f_{0}$ is the sum of two Dirac measures as above and a remaining part. But the value of $\delta$ obtained in this way, and $\varepsilon$, depend on $\left|w_{1}-w_{2}\right|$, and on how big a part of the initial data is concentrated in the two Dirac measures.

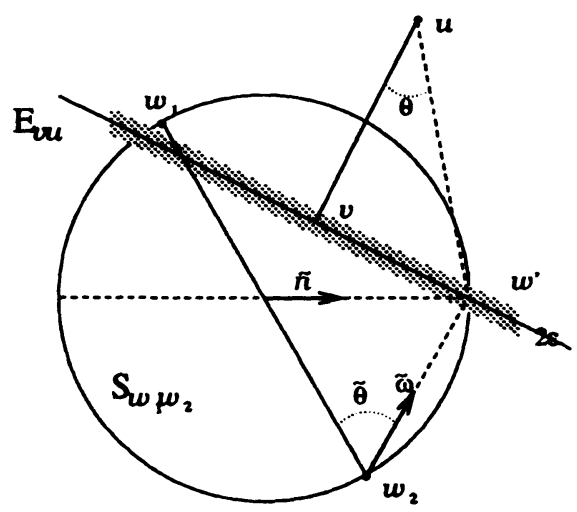

Fig. 1. Geometry for estimating the iterated gain term 


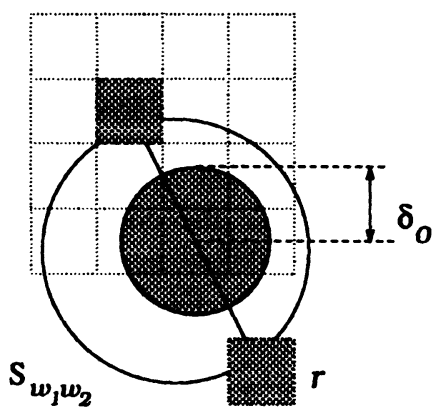

Fig. 2. The cubes $K_{1}$ and $K_{2}$

In order to find uniform estimates of $\delta$ and $\varepsilon$ (expressed in the mass, energy and entropy of the function $f$ ), we shall first prove that inside a large ball $|v|<\sqrt{3} R$, one can always find at least two small cubes (with side length $r$ ) separated by a distance $c r$ for a certain constant $c$, and such that these two cubes contain a part of the mass that can be uniformly estimated from below. The proof is then concluded by a convolution argument.

Let $K_{R}$ be the cube with side length $2 R$ centered at the origin, and subdivide this cube into $(2 R / r)^{3}$ cubes $K_{i}$ of length $r$. Denote the centre of the cube $K_{i}$ by $k_{i}$. If $R$ is sufficiently large, then

$$
\int_{K_{R}} f_{0}(v) d v>1 / 2
$$

Since the entropy is bounded, it is possible to compute a uniform upper bound on the mass contained in each subcube, and if $r$ is small enough,

$$
\int_{K_{i}} f_{0}(v) d v<\frac{1}{4 \cdot 3^{3}} \quad \text { for all } i
$$

This means that no set of 27 subcubes can contain more than half of the mass which is contained in $K_{R}$, and hence there are at least two subcubes, say $K_{1}$ and $K_{2}$, with centres separated by at least $2 \sqrt{3} r$, and such that

$$
\int_{K_{j}} f_{0}(v) d v \geqq \frac{1}{4(2 R / r)^{3}}, \quad j=1,2 .
$$

We claim that $\bar{v}=\left(k_{1}+k_{2}\right) / 2$ and $\delta_{0}=\left(\left|k_{1}-k_{2}\right|-\sqrt{6}\right) /(4 \sqrt{2}>(2 \sqrt{3}-\sqrt{6}) r \mid$ $(4 \sqrt{2})$ satisfy the statement of Lemma 3.1. To see that, let $w_{i} \in K_{i}, i=1,2$, and let $S_{w_{1}, w_{2}}$ be the sphere in which the segment $\overline{w_{1}, w_{2}}$ is a diagonal. A geometric argument shows that the ball with centre in $\bar{v}$ and radius $2 \delta_{0}$ lies entirely inside $S_{w_{1}, w_{2}}$ (see Fig. 2). Next, let $\chi_{j}$ be the characteristic function of $K_{j}$, let $\chi_{R}$ be the characteristic function of $K_{R}$, and write $f_{1}=f_{0} \chi_{1}, f_{2}=f_{0} \chi_{2}$, and $f_{3}=f_{0} \chi_{R}$. Then, with $A=c\left(1+(\sqrt{3} R)^{\beta}\right)$,

$$
f(v, t) \geqq \int_{0}^{t} \int_{0}^{\tau} e^{-A\left(t+\tau+\tau_{1}\right)} d \tau_{1} d \tau Q^{+}\left(f_{3}, Q^{+}\left(f_{1}, f_{2}\right)\right)(v) .
$$


Here $\tilde{G}_{t_{1}}^{t_{2}}(v)$ could be replaced by a plain exponential, since all the involved functions are supported in $K_{R} \subset\{|v|<\sqrt{3} R\}$, and then the $t$-integrals can be evaluated separately. For small $t$, the integrals in (3.7) become $t^{2} / 2-A t^{3}+\mathcal{O}\left(A^{2} t^{4}\right)$.

In order to simplify the estimate of the iterated gain term, we will assume that $h(\theta)$ is bounded from below by a constant $h_{0}$. This does not change the statement of the theorem, even though of course the constants are affected; however, it is important that $h(\theta)$ is bounded from below near $\theta=\pi / 4$ (this is in fact essential also in the proof of Theorem 1.1). As in the introductory discussion in this proof, we will use the Carleman representation to evaluate the "outer" gain term, and the usual representation for the "inner" one. In (3.4), $f$ is replaced by $f_{3}$, and $g$ by $Q^{+}\left(f_{1}, f_{2}\right)$, and, in order to make the notation slightly less awkward, we write $v^{\prime} \equiv u$, and $v_{1}^{\prime}=w$. We also write $f_{j}(v)=\int f_{j}\left(w_{j}\right) \delta\left(v-w_{j}\right) d w_{j}(j=1,2)$, and begin by evaluating the integral over $E_{v, v^{\prime}}$. This becomes

$$
\int_{\mathbb{R}^{3}} f_{1}\left(w_{1}\right) \int_{\mathbb{R}^{3}} f_{2}\left(w_{2}\right) \int_{E_{v, w}} Q^{+}\left(\delta_{1}, \delta_{2}\right)(w) \frac{h(\theta)}{(\cos \theta)^{\beta}} d E(w) d w_{2} d w_{1},
$$

where $\delta_{j}$ denotes the Dirac measure translated by $w_{j}$. Consider first the integral over $E_{v, u}$. Let $\Xi_{\varepsilon}$ denote the characteristic function of $\left\{w \mid \operatorname{dist}\left(w, E_{v, u}\right)<\varepsilon\right\}$. Then the $E_{v, u}$-integral is

$$
\begin{aligned}
& \lim _{\varepsilon \rightarrow 0} \frac{1}{2 \varepsilon} \int_{\mathbb{R}^{3}} \int_{\mathbb{R}^{3}} \int_{S^{2}} \delta_{1}\left(w^{\prime}\right) \delta_{2}\left(\tilde{w}^{\prime}\right) h(\tilde{\theta})|w-\tilde{w}|^{\beta} d \tilde{w} d \tilde{w} \frac{h(\theta)}{(\cos \theta)^{\beta}} \Xi_{\varepsilon}(w) d w \\
& \quad=\lim _{\varepsilon \rightarrow 0} \frac{1}{2 \varepsilon} \int_{\mathbb{R}^{3}} \int_{\mathbb{R}^{3}} \int_{S^{2}} \delta_{1}(w) \delta_{2}(\tilde{w})|w-\tilde{w}|^{\beta} h(\tilde{\theta}) \frac{h(\theta)}{(\cos \theta)^{\beta}} \Xi_{\varepsilon}\left(w^{\prime}\right) d \omega d \tilde{w} d w \\
& \quad=\left|w_{1}-w_{2}\right|^{\beta-2} \lim _{\varepsilon \rightarrow 0} \frac{1}{2 \varepsilon} \int_{S_{w_{1}, w_{2}}} \frac{h(\tilde{\theta})}{\cos \tilde{\theta}} \frac{h(\theta)}{(\cos \theta)^{\beta}} \Xi_{\varepsilon}\left(w^{\prime}\right) d \tilde{n} .
\end{aligned}
$$

The first of these equalities comes from the usual change of variables $d \tilde{w} d w \rightarrow$ $d \tilde{w}^{\prime} d w^{\prime}$, and the second one is a change of variables which can be found in Carleman; $S_{w_{1}, w_{2}}$ is the sphere which was defined earlier, and $d \tilde{n}$ denotes the surface measure on that sphere (see Fig. 1). The geometry gives estimates on the velocity differences (as well as on the angles $\theta$ and $\tilde{\theta}$, in the case where that is necessary; here it is not), and using also the conditions on $h(\theta)$, we find that the expression in (3.8) is bounded from below by

$$
\begin{aligned}
& \left|w_{1}-w_{2}\right|^{\beta-2} h_{0}^{2} \lim _{\varepsilon \rightarrow 0} \frac{1}{2 \varepsilon} \int_{S_{w_{1}, w_{2}}}(\cos \tilde{\theta})^{-1} \Xi_{\varepsilon}\left(w^{\prime}\right) d \tilde{n} \\
& \geqq 2 \pi\left|w_{1}-w_{2}\right|^{\beta-1} h_{0}^{2} \geqq 2 \pi \min \left((2 R)^{\beta-1},(2 r)^{1-\beta}\right) h_{0}^{2} \equiv \tilde{C} .
\end{aligned}
$$

Finally,

$$
\begin{aligned}
Q^{+}\left(f, Q^{+}(f, f)\right)(v) & \geqq \tilde{C} \int_{\mathbb{R}^{3}} f_{3}(u)|u-v|^{\beta-2} \int_{\mathbb{R}^{3}} f_{1}\left(w_{1}\right) \int_{\mathbb{R}^{3}} f_{2}\left(w_{2}\right) d w_{2} d w_{1} d u \\
& \geqq \tilde{C}(2 R)^{\beta-2} \frac{1}{2}\left(\frac{1}{4(2 R / r)^{3}}\right)^{2},
\end{aligned}
$$

and this, together with the estimate of the time integral, gives an estimate of $\varepsilon_{0}$. 
The following lemma is the analogue of Lemma 2.4 in Sect. 2.

Lemma 3.2. Suppose that there is a $\bar{v}$, with $|\bar{v}|<R$, such that $f(v)>\varepsilon$ for $|v-\bar{v}|<\delta$, then there is a constant $C$ such that

$$
Q^{+}(f, f)(v)>C \delta^{3+\beta} \gamma^{5 / 2} \varepsilon^{2},
$$

for all $v$ with $|v-\bar{v}|<\delta \sqrt{2}(1-\gamma)$. The constant $C$ depends only on $\beta$ and on the function $h(\theta)$.

Proof. The proof is a direct computation just as in the case of Lemma 2.4. Recall first that the operator is quadratic, positive and monotonous, and because of the Gallilean invariance of the collision operator, there is no loss of generality in assuming that $\bar{v}=0$. The Carleman representation of the gain term is useful also for establishing the scaling property:

$$
\begin{aligned}
Q^{+}(f, f)\left(\delta_{0} v\right) & =\int_{\mathbb{R}^{3}} f(u) \frac{1}{\left|u-\delta_{0} v\right|^{2-\beta}} \int_{E_{\delta_{0} v u}} f(w) h(\theta)(\cos \theta)^{-\beta} d E(w) d u \\
& =\delta_{0}^{3} \int_{\mathbb{R}^{3}} f\left(\delta_{0} u\right) \frac{1}{\delta_{0}^{2-\beta}|u-v|^{2-\beta}} \int_{E_{\delta_{0} \delta_{0} u}} f(w) h(\theta)(\cos \theta)^{-\beta} d E(w) d u \\
& =\delta_{0}^{3} \int_{\mathbb{R}^{3}} f\left(\delta_{0} u\right) \frac{1}{\delta_{0}^{2-\beta}|u-v|^{2-\beta}} \delta_{0}^{2} \int_{E_{v u}} f\left(\delta_{0} w\right) h(\theta)(\cos \theta)^{-\beta} d E(w) d u \\
& =\delta_{0}^{3+\beta} Q^{+}\left(f\left(\delta_{0} \cdot\right), f\left(\delta_{0} \cdot\right)\right)(v) .
\end{aligned}
$$

The angle $\theta$ is unchanged, since all variables are simply rescaled. From now on in this proof, let $f$ be the characteristic function of the set $\{v ;|v|<1\}$. By symmetry, we can assume that $v=(0,0, z)$, with $1 \leqq z<\sqrt{2}$. Then, using polar coordinates for $u$, taking $v$ as the origin (so that $d u=r^{2} \sin \alpha d \alpha d r$ ), gives

$$
\begin{aligned}
Q^{+}(f, f)(v) & =\int_{\mathbb{R}^{3}} f(u) \frac{1}{|u-v|^{2-\beta}} \int_{E_{v, u}} f(w) \frac{h(\theta)}{(\cos \theta)^{\beta}} d E(w) d u \\
& =2 \pi \int_{0}^{\pi} \int_{0}^{\infty} f(u) \frac{1}{r^{2-\beta}} \int_{E_{v u}} f(w) \frac{h(\theta)}{(\cos \theta)^{\beta}} d E(w) \sin \alpha d \alpha r^{2} d r .
\end{aligned}
$$

The integrand is non-zero only when $|u| \leqq 1$ and $|w| \leqq 1$, i.e. when $\arccos (1 / z) \leqq$ $\alpha \leqq \arcsin (1 / z)$, and $z \cos \alpha-\sqrt{1-z^{2} \sin ^{2} \alpha} \leqq r \leqq z \cos \alpha+\sqrt{1-z^{2} \sin ^{2} \alpha}$ hold simultaneously. By a change of variables, $y=z \cos \alpha$, and by replacing $h(\theta)(\cos \theta)^{-\beta}$ with $h_{0}$, we find that (see Fig. 3)

$$
Q^{+}(f, f)(v) \geqq \frac{2 \pi^{2} h_{0}}{z} \int_{\sqrt{z^{2}-1}}^{1} \int_{y-\sqrt{1-z^{2}+y^{2}}}^{y+\sqrt{1-z^{2}+y^{2}}} r^{\beta} d r\left(1-y^{2}\right) d y,
$$

and it only remains to estimate the behaviour near $z=\sqrt{2}$. Let $x=1-y$ and write $z=\sqrt{2}(1-\gamma)$. The integral over $r$ then becomes $2 \sqrt{4 \gamma-2 x}+\mathcal{O}\left(\gamma^{3 / 2}\right)$, and so the 


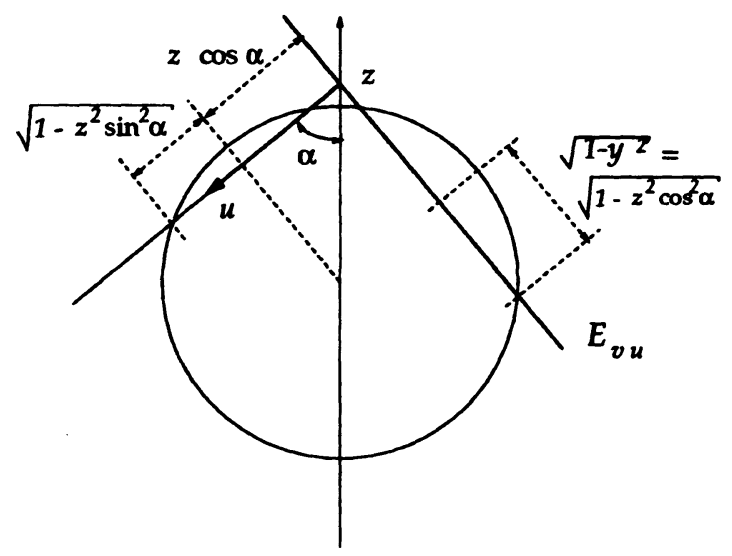

Fig. 3. The gain term vanishes when the ball lies inside the corner

right-hand side of (3.9) becomes (note that also $x=\mathcal{O}(\gamma)$ )

$$
\begin{aligned}
& 8 \pi^{2} h_{0}(1+\mathcal{O}(\gamma)) \int_{0}^{2 \gamma+\mathcal{O}\left(\gamma^{2}\right)}\left(\sqrt{2 \gamma-x}+\mathcal{O}\left(\gamma^{3 / 2}\right)\right) x(1+\mathcal{O}(\gamma)) d x \\
& =8 \pi^{2} h_{0}(1+\mathcal{O}(\gamma))(2 \gamma)^{5 / 2} \int_{0}^{1+\mathcal{O}(\gamma)}(\sqrt{1-x}+\mathcal{O}(\gamma))(x+\mathcal{O}(\gamma)) d x \\
& =\frac{64 \sqrt{2} \pi^{2}}{15} \gamma^{5 / 2}+\mathcal{O}\left(\gamma^{7 / 2}\right) .
\end{aligned}
$$

The proof of Theorem 1.1 can now be completed by using the iteration argument from Sect. 2. The starting point is Lemma 3.1, which provides a ball $|v-\bar{v}|<\delta_{0}$ in which $f\left(v, t_{0}\right)>\varepsilon_{0}$. The main difference between this and the corresponding result for the Kac equation, is that here $\varepsilon_{0}=\mathcal{O}\left(t_{0}^{2}\right)$.

The iteration procedure itself is slightly more complicated here, mainly because of the unbounded loss term. In the first step,

$$
f\left(v, t_{0}+t_{1}\right) \geqq \int_{t_{1}}^{t_{0}+t_{1}} G_{\tau}^{t_{0}+t_{1}} Q^{+}\left(G_{t_{0}}^{\tau} f\left(\cdot, t_{0}\right), G_{t_{0}}^{\tau} f\left(\cdot, t_{0}\right)\right)(v) d \tau .
$$

At this point, we are only interested in the behaviour of $f$ and $Q^{+}$in or near the given ball, and so, for all relevant velocities,

$$
G_{\tau_{1}}^{\tau_{2}} \geqq e^{-\left(\tau_{2}-\tau_{1}\right) c\left(1+2|\bar{v}|^{\beta}+2^{1+\beta / 2} \delta_{0}^{\beta}\right)} \equiv e^{-\left(\tau_{2}-\tau_{1}\right) \bar{c}} .
$$

With this expression inserted into (3.10), the time dependent factors can be taken out of $Q^{+}$, and the time integral can be carried out. The result of that is

$$
\begin{aligned}
f\left(v, t_{0}+t_{1}\right) & \geqq e^{-t_{1} \bar{c} \frac{1-e^{-t_{1} \bar{c}}}{\bar{c}}} Q^{+}(f, f)(v) \geqq t_{1} e^{-t_{1} 2 c\left(1+2|\bar{v}|^{\beta}+2^{1+\beta / 2} \delta_{0}^{\beta}\right)} C \delta_{0}^{3+\beta} \gamma_{1}^{5 / 2} \varepsilon_{0}^{2} \\
& \geqq t_{1} e^{-2 c t_{1} 2^{\beta / 2} \delta_{0}^{\beta}} C \delta_{0}^{3+\beta} \gamma_{1}^{5 / 2} \varepsilon_{0}^{2},
\end{aligned}
$$


and this holds for all $|v-\delta|<\sqrt{2}\left(1-\gamma_{1}\right) \delta_{0}$. In the last step, we made the assumption that $t_{1}$ is smaller than some given constant, and included the part of the exponential that contains $1+2|\bar{v}|$ in $C$. The estimate after the second step in the iteration in this case is (cf. Sect. 2)

$$
\begin{aligned}
f\left(v, t_{0}+t_{1}+t_{2}\right) \geqq & \left(t_{1} e^{-t_{1} c 2^{1+\beta / 2} \delta_{0}^{\beta}} C \delta_{0}^{3+\beta} \gamma_{1}^{5 / 2} \varepsilon_{0}^{2}\right)^{2} \\
& \cdot C t_{2} e^{-t_{1} c 2^{1+2 \beta / 2} \delta_{0}^{\beta}}\left(2^{1 / 2}\left(1-\gamma_{1}\right) \delta_{0}\right)^{3+\beta} \gamma_{2}^{5 / 2},
\end{aligned}
$$

for $|v-\bar{v}|<2^{2 / 2}\left(1-\gamma_{1}\right)\left(1-\gamma_{2}\right) \delta_{0}$, and after the $n^{\text {th }}$ step,

$$
\begin{aligned}
f\left(v, t_{0}+\cdots+t_{n}\right)> & \varepsilon_{0}^{2^{n}}\left(C \delta_{0}^{3-\beta}\right)^{2^{n}-1} \cdot\left(2^{1 / 2}\left(1-\gamma_{1}\right)\right)^{(3+\beta) 2^{n-1-1}} \\
& \cdots \cdot\left(2^{k / 2}\left(1-\gamma_{1}\right) \cdots \cdot\left(1-\gamma_{k}\right)\right)^{(3+\beta) 2^{n-1-k}} \\
& \cdots \cdot\left(t_{1} \gamma_{1}^{5 / 2}\right)^{2^{n-1}} \cdots \cdot\left(t_{k} \gamma_{k}^{5 / 2}\right)^{2^{n-k}} \cdots \cdots \cdot\left(t_{n} \gamma_{n}^{5 / 2}\right)^{2^{0}} \\
& \cdot e^{-c t_{1} \delta_{0}^{\beta} 2^{1+\beta / 2} 2^{n-1}} \cdots \cdot e^{-t_{k} c \delta_{0}^{\beta} 2^{1+\beta k / 2} 2^{n-k}} \cdots \cdots \cdot e^{-t_{n} c \delta_{0}^{\beta} 2^{1+\beta n / 2} 2^{n-n}}
\end{aligned}
$$

for $|v|<\delta_{0} 2^{n / 2}\left(1-\gamma_{1}\right) \cdots\left(1-\gamma_{n}\right)$. This differs from (2.13) only in the exponential factors at the end, and therefore the proof of Theorem 1.1 is complete once one has checked that that product converges. And this holds with the same choice of $t_{k}$ and $\gamma_{k}$ as in Sect. 2 if $\beta<2$, which includes all relevant cases.

Acknowledgements. B.W. would like to express his sincere gratitude to E. Gabetta and G. Toscani for their kind hospitality while he was visiting the University of Pavia. Financial support from the National Council for Research of Italy (Gruppo Nazionale per la Fisica Matematica del CNR) for the visit in Italy, and from the Swedish Research Council for Natural Sciences for the academic year 94-95 are gratefully acknowledged.

\section{References}

[A1] Arkeryd, L.: On the Boltzmann equation. Arch. Rational Mech. Anal. 34, 1-34 (1972)

[A2] Arkeryd, L.: $L^{\infty}$-estimates for the space homogeneous Boltzmann equation. J. Statist. Phys. 31, 347-361 (1982)

[Bo] Bobylev, A.V.: The theory of the nonlinear spatially uniform Boltzmann equation for Maxwellian molecules. Sov. Scient. Rev. c 7, 111-233 (1988)

[Ca1] Carleman, T.: Sur la solution de l'equation intégrodifferential de Boltzmann. Acta Math. 60, 91-146 (1933)

[Ca2] Carleman, T.: Problèmes mathématiques dans la théorie cinétique des gaz. Uppsala: Almqvist \& Wiksell, 1957

[C-C] Carlen, E.A., Carvalho, M.C.: Entropy production estimates for Boltzmann equations with physically realistic collision kernels. J. Statist. Phys. 74, Nos. 3/4, 743-782 (1994)

[Ce] Cercignani, C.: The theory and application of the Boltzmann equation. Applied Mathematical Sciences 67, New York: Springer, 1988

[D1] Desvillettes, L.: Entropy dissipation rate and convergence in kinetic equations. Commun. Math. Phys. 123, 687-702 (1989)

[D2] Desvillettes, L.: On the regularizing properties of the non cut-off Kac equation. Commun. Math. Phys. 168, 417-440 (1995)

[D-L] DiPerna, R., Lions, P.-L.: On the Cauchy problem for the Boltzmann equation; global existence and weak stability. Ann. Math. 130, 321-366 (1989)

[G-P] Gabetta, E., Pareschi, L.: About the non cut-off Kac equation: Uniqueness and asymptotic behaviour. Preprint 911/94, Istituto di Analisi Numerica del CNR, Pavia (1994) 
[G-T] Gabetta, E., Toscani, G.: On convergence to equilibrium for Kac's caricature of a Maxwell gas. J. Math. Phys. 35, 1 (1994)

[K] Kac, M.: Probability and related topics in the physical sciences. New York: Interscience Publishers, 1959

[M] McKean, H.P., Jr.: Speed of approach to equilibrium for Kac's caricature of a Maxwellian gas. Arch. Rational Mech. Anal. 21, 343-367 (1966)

[P-W] Pulvirenti, A., Wennberg, B.: Lower bounds for the solution to the Kac and the Boltzmann equation. To appear in Transp. Theory Stat. Phys.

[W] Wennberg, B.: On an entropy dissipation inequality for the Boltzmann equation. C.R. Acad. Sci. Paris t. 315, Série I, 1441-1446 (1992)

Communicated by J.L. Lebowitz 